prison, a lack of officers precipitated a 'dangerous environment', where safety concerns constrained patient access. Within the high performing prison, clinic set-up fostered interaction between health workers and officers. Social relationships developed, with staff occupying a liminal space, facilitating the development of informal strategies that maximised testing.

The success reported by some London prisons is a vulnerable one. Until wider structural pressures are addressed and opt-out testing securely embedded, staff change risks programme disintegration. Results suggest there is opportunity for innovation within the liminal space between the prison and healthcare, but formalisation of roles within this space is recommended. Further work is required to ensure London prisons are on track to facilitate elimination targets.

\section{P24 STUDY PROTOCOL: A CRITICAL COMMUNICATIVE EVALUATION OF MATERNAL CARE PATHWAYS FOR FGM IN LOTHIAN}

Amanda Di Rosa. Queen Margaret University, Edinburgh, UK

\subsection{6/bmjopen-2019-QHRN.59}

Background For expectant mothers with female genital mutilation (FGM), relevant care pathways are critical to a positive maternal care experience. Women with FGM may require a range of unique support, from mental health counselling to antenatal vaginal surgery. ${ }^{5}$ Challenged by evidence of substandard patient care, poor staff knowledge, and an increase in FGM-affected populations across Scotland, NHS Lothian established a specialist care team and training for FGM in 2015. ${ }^{1,3,4}$ This protocol presents an adaptation of the critical communicative methodology (CCM) to understand NHS staff and community views on the impact of these developments, and how the system can continue to improve.

Objective To present a publicly led strategy for the evaluation of training and maternal care for women with FGM in Lothian.

Methods CCM provides a useful tool for researchers to avoid the disadvantages of excluding the public from healthcare research and development. ${ }^{2}$ The method treats the contributions of researchers and the community equally, resulting in actionable and relevant evidence-based priorities for improvement. This protocol details our use of CCM's approach to include relevant, diverse voices (the NHS Lothian FGM team, midwives, and new mothers) in every step of the research process. In-depth interviews partner researchers and individuals in an exploration of existing research evidence and their impressions of the new maternal strategy for FGM. Democratic discussion groups then analyze these views to determine recommendations for future maternal training and care for women with FGM. Ethical approval for this study was obtained from NHS South East Scotland.

Conclusion This protocol should be of interest to those seeking to apply methods with an emphasis on social justice, which recognize the agency of the public to contribute to the healthcare strategies affecting their communities.

\section{REFERENCES}

1. Baldeh F. Obstetric care in Scotland: The experience of women who have undergone Female Genital Mutilation (FGM) MSc edn, 2013. Queen Margaret University.

2. Gómez J, Latorre A, Sánchez M, Flecha R. Metodología Comunicativa Crítica [Critical Communicative Research], 2006. Barcelona, Spain: Hipatia.

3. Moxey JM, Jones LL. A qualitative study exploring how Somali women exposed to female genital mutilation experience and perceive antenatal and intrapartum care in England. BMJ Open 2016;6(1):e009846.

4. Scottish Refugee Council (SRC). Tackling female genital mutilation in Scotland: A Scottish model of intervention, 2014. [Online] Available at: http://www.scottish refugeecouncil.org.uk/assets/0000/9061/FGM_Report_FINAL_A4portrait. pdf [Accessed 19 Sept. 2018].

5. World Health Organization (WHO). Health risks of Female Genital Mutilation (FGM), 2018. [online] Available at: http://www.who.int/reproductivehealth/topics/ fgm/health_consequences_fgm/en/[Accessed 19 Sept. 2018].

\section{P25 BALANCING PERSPECTIVES ON INTERVENTION FEASIBILITY: USING STAKEHOLDER VIEWS IN DECISION-MAKING}

${ }^{1}$ Jackie Fox, ${ }^{2}$ Lena-Karin Erlandsson, ${ }^{1}$ Agnes Shiel. 'National University of Ireland Galway, Galway, UK; ${ }^{2}$ Lund University, Lund, Sweden

\subsection{6/bmjopen-2019-QHRN.60}

Background Anxiety and stress are prevalent in general practice and primary care settings, particularly among women. The 'Redesigning Daily Occupations' (ReDO) programme is a manualised occupational therapy-led intervention designed to improve balance in daily life and reduce stress. A pilot study aiming to explore the feasibility of implementing ReDO in primary care settings was carried out. Despite predicted demand, recruitment was slower than anticipated.

Objectives

- To understand the feasibility and acceptability of ReDO from the perspective of stakeholders.

- To understand the study processes better.

- To use this information in decision-making to plan future intervention evaluation/development.

Methods Six women diagnosed with anxiety or stress-related conditions were recruited to take part in the 10 week group programme via their general practitioner. Qualitative interviews were completed with five group participants, two group facilitators, and nine general practitioners $(n=17)$. The data analysis was informed by principles of qualitative evaluation research where the focus is on achieving practical, actionable understandings of real-world issues in context. ${ }^{1}$

Results Results demonstrated conflicting perspectives on the feasibility of ReDO. The participants noted changes in their daily lives in mental health and daily functioning and recommended longer interventions. In contrast, the length of the intervention was balanced with a heavy clinical workload for the occupational therapists, while general practitioners felt the length of the programme restricted study recruitment. The occupational therapy programme facilitators and participants contributed to understanding how changes had taken place within the context of group dynamics adding to insight into who the intervention might best be suited to.

Conclusions The qualitative approach contributed greatly to decision-making. The programme will be delivered again in 2019 with small changes to the inclusion/exclusion criteria and recruitment strategy. Future research will explore the 
mechanism of change as understood by participants and facilitators within this intervention.

\section{REFERENCE}

1. Patton M. Qualitative research and evaluation methods: Integrating theory and practice (4th Edition), 2015. Thousand Oaks, CA: SAGE Publications, Inc.

\section{P26 UNDERSTANDING THE USE OF DATA FOR IMPROVEMENT}

Duncan Wagstaff, Cecilia Vindrola, Naomi Fulop, Ramani Moonesinghe. University College London, London, UK

\subsection{6/bmjopen-2019-QHRN.61}

Background Using data for improvement is a national policy priority. ${ }^{1}$ However, despite the considerable resources required to collect data for national clinical audits, their information is rarely used effectively to improve quality. ${ }^{2}$ Barriers lie with the collection, analysis and feedback of these data, as well as with the capability, capacity and motivation of local teams to use them for improvement. ${ }^{3}$ The perioperative setting encompasses the period before, during and after surgery and represents an increasingly recognised healthcare sector. ${ }^{4}$ A scoping review we have conducted has confirmed that national clinical audit data is used inconsistently for local improvement of perioperative services. ${ }^{5}$ The new national Perioperative Quality Improvement Programme (PQIP) aims to facilitate this use of data to improve perioperative services in the NHS. ${ }^{6}$

Aim This study seeks to explore whether, how, and in which contexts, perioperative data are currently used for quality improvement, and how this is affected by PQIP.

Methods A mixed-methods approach has been adopted comprising a national survey and qualitative fieldwork. The survey is gathering multidisciplinary staff experiences of using perioperative data and their perceptions of contextual factors. Findings from the survey will be triangulated with qualitative fieldwork in two case study hospitals, before and after they engage with PQIP. Furthermore, local understanding of PQIP's programme theory in these case study hospitals will be compared with that of the central PQIP project team to help understand any implementation gaps.

Results Data collection and analysis remains ongoing but will be available by March 2019.

Conclusions Using data for improvement in the NHS has often been an aspiration rather than an achievement due to difficulties posed by defining, measuring and improving quality. This study seeks to understand whether a theoretically derived program can overcome these barriers in the perioperative setting.

\section{REFERENCES}

1. National Improvement and Leadership Development Board. Developing People Improving Care, 2016.

2. Allwood D. Engaging Clinicians in Quality Improvement through National Clinical Audit. Rep to Health Qual Improv Partnersh. 2014;2014(October).

3. The Royal College of Physicians and the Healthcare Quality Improvement Partnership. Unlocking the Potential Supporting Doctors to Use National Clinical Audit to Drive Improvement; 2018.

4. The Royal College of Anaesthetists. Perioperative Medicine the Pathway to Better Surgical Care; 2015.

5. University of York, Centre for Reviews and Dissemination. PROSPERO. https:// www.crd.york.ac.uk/prospero/. CRD42018092993.

6. www.pqip.org.uk.

\section{P28 THE SOCIAL INVISIBILITY OF MENTAL HEALTH: UNDERSTANDING SOCIAL EXCLUSION THROUGH PLACE \& SPACE}

${ }^{1}$ Evangelia Chrysikou, ${ }^{2}$ Elefteria Savwopoulou, ${ }^{3}$ Efstathia Kostopoulou, ${ }^{4}$ Naaheed Mukadam, ${ }^{5}$ Ioanna Tsimopoulou, ${ }^{6}$ Sarah Pickering, ${ }^{7}$ Ava Fatah Gen Schieck. ${ }^{1}$ The Bartlett Real Estate Institute, University College London (UCL), London, UK; ${ }^{2}$ SynThesis Architects, London, UK; ${ }^{3}$ University College London, London, UK; ${ }^{4}$ University College London, Division of Psychiatry, London, UK; ${ }^{5}$ Camden and Islington NHS Foundation Trust, London, UK; ${ }^{6}$ Slade School of Fine Art UCL, London, UK; ${ }^{7}$ The Bartlett School of Architecture, UCL, London, UK

\subsection{6/bmjopen-2019-QHRN.62}

Background Mental health is considered the Cinderella of any healthcare system. There is a European target that the 5\% of the healthcare service budget goes to mental health and most countries have reached that target. Yet, this might not be enough to cover the inequity between health and mental health provision.

Aim 'The social invisibility of mental health facilities' is a multi-disciplinary, innovative, research-through-arts project, involving a School of Architecture, a Division of Psychiatry and a School of Art. The aim is to detect if there are elements demonstrating inequality demonstrated from place and space related to the facility provision.

Methods It compares healthcare vs mental health facilities of the same catchment area, raising awareness of inequalities between the two and the social exclusion of mentally ill people through a visual, multimedia perspective. It juxtaposes (mental) healthcare facilities in terms of access, condition and status compared to their surroundings. The exhibits were created from both art and architectural schools postgraduate students. The exhibition took place close to Bentham's auto-icon, the designer of Panopticon custodial facility, demonstrating inverse links between his Panopticon, and the concealment/invisibility that NIMBYism produces towards the mentally ill that resulted in their exclusion, within deprived, under-funded, isolated facilities in the community'.

Conclusions The exhibition with the satellite actions, such as the mapping of the facilities and the picture-rich book demonstrated the under-budgeting of mental health facilities and their stigmatization as expressed by the centrality of locations and the overall projected image of the building exteriors. This outlined the path for integrated, transdisciplinary research in the future involving architecture, arts and psychiatry.

\section{P29 THE ELECTRONIC CAPTURE OF PATIENT-REPORTED OUTCOMES IN TRAUMA RESEARCH: VIEWS FROM THE FIELD}

${ }^{1}$ Christel McMullan, ${ }^{1}$ Grace Turner, ${ }^{1}$ Derek Kyte, ${ }^{1}$ Anita Slade, ${ }^{1}$ Ameeta Retzer, ${ }^{2}$ Tony Belli, ${ }^{2}$ Karen Piper, ${ }^{1}$ Mel Calvert. 'Institute of Applied Health Research, University of Birmingham, Birmingham, UK; ${ }^{2}$ University Hospital Birmingham NHS Foundation Trust, Birmingham, UK

\subsection{6/bmjopen-2019-QHRN.63}

Background The numbers of major trauma and traumatic brain injury (TBI) survivors are increasing. Finding efficient ways to capture the impact of symptoms on their quality of 